\title{
Complex Emotional Intelligence Learning Using Deep Neural Networks (Student Abstract)
}

\author{
Belainine Billal, Sadat Fatiha, Lounis Hakim \\ ${ }^{1}$ University of Quebec in Montreal \\ 2098 Rue Kimberley, Montreal, QC H3C 3P8 \\ Quebec, Canada \\ belainine.billal@courrier.uqam.ca
}

\begin{abstract}
Emotion recognition and mining tasks are often limited by the availability of manually annotated data. Several researchers have used emojis and specific hashtags as forms of training and supervision.

This research paper proposes a new textual and social corpus, the corpus labeled using basic emotions following Plutchik's theory. Thus, This paper propose a first study for the representation and interpretation of complex emotional interactions, using deep neural networks.
\end{abstract}

\section{Introduction}

Several researchers proposed their approaches to express and recognize emotions, according to: (1) The intensity or valence, arousal, etc.(Posner, Russell, and Peterson, 2005). (2) The categorical model of the basic emotions. This model type proposes the detection of basic emotions that are considered as atomic and irreducible, or following a model established by (Plutchik, 1980), which represents a complex emotion as a combination of several basic emotions (De Bonis, 1996). The present research paper describes a study designed to detect complex emotions according to the model proposed by Plutchik. Our main contributions in this study are described as follows: (1) Introduction of a language model transfer to a multi-label classifier based on the architecture of the transformer decoder. (2) Introduction of a formal method for reading and interpreting complex emotions based on basic emotion vectors.

\section{The Proposed Approach}

Our proposed approach consists of creating an emotional classifier able to detect the eight basic emotions according to the Plutchik model. This classifier use conflicting emotion representation as an implicit rule, summed up by three main phases. First, we proceed by collecting the annotated data for the construction of corpus, by filtering and mixing several types of corpora in order to collect the eight of basic emotions. The second phase is the detection of emotional vector using four dimensions space. The third phase consists of interpreting and reading complex emotions.

Copyright (C) 2020, Association for the Advancement of Artificial Intelligence (www.aaai.org). All rights reserved.

\section{Corpus :}

Our training corpus is based on a combination and filtering of several datasets from several sources. We have broken down the complex emotions that existed in our corpus as: 1) Love $=$ Joy + Trust. 2) Shame $=$ Fear + Disgust. 3) Guilt $=$ Fear + Joy .

Table 1: Sources of all used Corpora

\begin{tabular}{|c|c|}
\hline Dataset & Labels \\
\hline EmoTxt $^{1}$ & $\begin{array}{l}\text { joy, anger, sadness, love, surprise, } \\
\text { fear }\end{array}$ \\
\hline PsychExp $^{2}$ & $\begin{array}{l}\text { joy, fear, anger, sadness, disgust, } \\
\text { shame, guilt }\end{array}$ \\
\hline DailyDialog $^{3}$ & $\begin{array}{l}\text { no emotion, anger, disgust, fear, } \\
\text { happiness, sadness, surprise }\end{array}$ \\
\hline $\begin{array}{l}\text { NRC_Emotion_Lexicon_ v0.92 } \\
4 \text { emotion_proposition_store } 5\end{array}$ & $\begin{array}{l}\text { joy, fear, disgust, anger, sadness, } \\
\text { surprise, trust and anticipation }\end{array}$ \\
\hline WordNetAffect (Valitutti, 2004) & $\begin{array}{l}\text { joy, fear, disgust, anger, sadness, } \\
\text { surprise, trust and anticipation }\end{array}$ \\
\hline
\end{tabular}

These data contain the eight basic emotions according to Plutchik's theory. In addition, there are three complex emotions that generally represent the complex emotions of our model.

\section{Detecting and Reading complex emotions.}

Our problem transformed into a multi-label learning problem where each of the four dimensions is represented by a label with three values $(1.0,-1)$, each label detected by a Sequence to Vector model (Seq2vec). we mark 1 if the emotion is present and -1 if the inverse of the emotion is present. If the emotion with its inverse is absent, we mark 0 . We use transfer learning of a DeepMoji model.We propose to difine the number of dimensions of our basic space is four pairs of emotions and is formally defined by the base $\mathrm{B}=((\mathrm{J}) \mathrm{y}$, Sadness), (Trust, Disgust), (Fear, Anger), (Surprise, Anticipation)). Table 2 shows a representation of primary complex emotions (Primary dyads).

Table 2 is used as a transition matrix $W$ to detect complexes emotions for primary dyads.

\footnotetext{
${ }^{1}$ https://github.com/collab-uniba/EMTk

${ }^{2}$ https://github.com/bfelbo/DeepMoji/tree/master/data

${ }^{3}$ http://yanran.li/dailydialog

${ }^{4}$ Lexicon of the NRC Word Emotion Association.
} 
Table 2: Combinations of 2 adjacent emotions that make the primary dyads in 4 dimensions.

\begin{tabular}{|l|l|l|l|l|}
\hline $\begin{array}{l}\text { Advanced Emo- } \\
\text { tions Primary } \\
\text { Dyad }\end{array}$ & $\begin{array}{l}\text { Anticipation- } \\
\text { Surprise }\end{array}$ & $\begin{array}{l}\text { Joy- } \\
\text { Sadness }\end{array}$ & $\begin{array}{l}\text { Trust- } \\
\text { Disgust }\end{array}$ & $\begin{array}{l}\text { Fear- } \\
\text { Anger }\end{array}$ \\
\hline \hline Optimism & 1 & 1 & 0 & 0 \\
\hline Love & 0 & 1 & 1 & 0 \\
\hline Submission & 0 & 0 & 1 & 1 \\
\hline Apprehension & -1 & 0 & 0 & 1 \\
\hline Disappointment & -1 & -1 & 0 & 0 \\
\hline Remord & 0 & -1 & -1 & 0 \\
\hline Contempt & 0 & 0 & -1 & -1 \\
\hline Aggressiveness & 1 & 0 & 0 & -1 \\
\hline
\end{tabular}

In equations 1 and 2, we show how one can detect the presence of a complex emotion by multiplying the matrix $W$ by the vector $V$, which represents the coordinates of the emotions in our vector space (equation 1). We use same process to generate the transition matrix of Secondary dyads and Tertiary dyads.

$$
\begin{gathered}
\underset{\text { Primary Dyad }}{S}=\underset{\text { Primary Dyad }}{W} V \\
\left\{\begin{array}{c}
\text { Emotion complex }=\underset{i}{\operatorname{argmax}}\left(S_{i}\right) \\
\text { and } \\
S_{i} \geq 1
\end{array}\right.
\end{gathered}
$$

$\mathrm{i} \in($ Optimism $=0$, Love $=1$, Submission $=2$, Alarm $=3$, Disappointment=4,Contemptment $=5$, Remord=6, Aggressive ness=7)

The result for the complex emotion obtained should be the result that maximizes a component of the vectors.

Equation 2 presents our objective function for reading the complex emotion. The complex emotions generated by the index $i$ correspond to the emotions in the transition matrix $W$ given in table 2 .

\section{Experiments and Analysis}

We conducted one sets of experiments on four models. Each model has three classes $(1,-1,0)$ that reflect one dimension of the emotion and its inverse, as well as the absence of both. Thus, four classifiers are proposed, as follows: Joy/Sadness , Trust/Disgust, Anticipation/Surprise, Anger/Fear.

The first set of experiments was based on the eight emotions as proposed in Plutchik's model. In order to represent those emotions well, we converted the complex emotions that exist in the corpus using a composition of the basic emotions.

Table 3: Results of Recall, Precision, F-score for different classifiers after using transfer learning.

\begin{tabular}{|l|l|l|l|l|l|}
\hline Axis Emotions & Recall & Precis & F1 & Macro F1 & $\begin{array}{l}\text { Exact } \\
\text { Match }\end{array}$ \\
\hline \hline joy/sadness & 0.56 & 0.44 & 0.49 & 0.54 & 0.43 \\
anger/fear & 0.61 & 0.56 & 0.58 & & \\
surprise/anticipation & 0.55 & 0.51 & 0.52 & & \\
trust/disgust & 0.63 & 0.59 & 0.57 & & \\
\hline
\end{tabular}

The table 4 presents a comparison with the state of the art for public datasets that contains some complex emotions. EmoTxt with a test that contains 200 instances of the labels
Table 4: Results based on Exact Match, F-score for Love, Guilt and Shame classification after using transfer learning.

\begin{tabular}{|l|l|l|l|}
\hline Model & Complex Emotion & Average-F1 & Exact Match \\
\hline \hline \multirow{3}{*}{ Our Model } & Love (Joy + Trust) & $\mathbf{0 . 5 8}$ & 0.52 \\
\cline { 2 - 4 } & $\begin{array}{l}\text { Shame (Fear + Dis- } \\
\text { gust) }\end{array}$ & 0.54 & 0.53 \\
\cline { 2 - 4 } & Guilt (Fear + Joy) & $\mathbf{0 . 5 4}$ & 0.51 \\
\hline \hline Model & Complex Emotion & F1 & Accuracy \\
\hline \hline \multirow{2}{*}{ DeepMoji( PsychExp) } & Shame & 0.56 & $\mathbf{0 . 5 9}$ \\
\cline { 2 - 4 } & Guilt & $\mathbf{0 . 5 4}$ & $\mathbf{0 . 6 0}$ \\
\hline Model & Complex Emotion & F1 & Accuracy \\
\hline \hline \multirow{2}{*}{ DeepMoji (PsychExp + EmoTxt) } & Love & 0.57 & $\mathbf{0 . 6 3}$ \\
\cline { 2 - 4 } & Shame & $\mathbf{0 . 5 3}$ & 0.58 \\
\cline { 2 - 4 } & Guilt & $\mathbf{0 . 5 1}$ & 0.58 \\
\hline
\end{tabular}

'Love' and PsychExp with a test that contains 264 instances of labels Guilt and 427 Shame. then we used the DeepMoji model Felbo et al. with a PsychExp dataset, but for the second we added the Love label to the model after training with PsychExp and EmoTxt dataset. The Love label represents the Joy + Trust detection found in the Primary Dyads, the Shame label represents the 'Fear + Disgust' detection found in the Secondary Dyads, the Guilt label represents the 'Fear + Joy' detection that is in the Tertiary Dyads.

By analyzing our results in table 4 , we can notice a slight difference between the different experiments.

The average F1 score of our model for label Guilt is equal to the F1 score of the experiment done by the DeepMoji model (PsychExp), but the DeepMoji model accuracy exceeds Exact match of our model by 0.07 , because the exact match means that both labels detect it at the same time.

Our model is better in terms of average F1 score for the label Love (Trust + Joy) compared to the DeepMoji (PsychExp + EmoTxt) model F1-score which contains the Love label.

\section{Conclusions and perspectives}

This research paper presents a novel approach for the detection of complex emotions, according to the Plutchik model and using multi-label classifiers. These classifiers are divided into 4 multiclass classifiers. The main contribution is the representation of complex emotions according to the Plutchik theory, in a vector space with four axes.

Due to the nature of social media text, some emotions, such as fear and disgust, are under-represented in the datasets. Also, other emotions, like anticipation, are absent.

\section{References}

De Bonis, M. 1996. Connaître les émotions humaines, volume 212. Editions Mardaga.

Felbo, B.; Mislove, A.; Sogaard, A.; Rahwan, I.; and Lehmann, S. Using millions of emoji occurrences to learn any-domain representations for detecting sentiment, emotion and sarcasm.

Plutchik, R. 1980. Emotion: A psychoevolutionary analysis. Nueva York: Harper and Row.

Posner, J.; Russell, J. A.; and Peterson, B. S. 2005. The circumplex model of affect: An integrative approach to affective neuroscience, cognitive development, and psychopathology. Development and Psychopathology 17(3):715-734.

Valitutti, R. 2004. Wordnet-affect: an affective extension of wordnet. In In Proceedings of the 4th International Conference on Language Resources and Evaluation, 1083-1086. 